since the symposium, the production of these volumes two years after the event raises once again the wisdom of publishing such conference proceedings in full, if at all. Certainly the high quality of the printing and binding suggests a permanence which the contents are unlikely to achieve.

A. E. J. EgGLeton

\section{ADAPTATIONS OF EGO-IDENTITY}

\section{Insight and Responsibility}

Lectures on the Ethical Implications of Psychoanalytic Insight. By Erik H. Erikson. Pp. 256. (London: Faber and Faber, Ltd., 1966.) 30s. net.

Prof. ErIkson had his psychoanalytic training in Vienna, and in his early work treating children there he had the guidance of Anna Freud and August Aichhorn. After he emigrated to the United States in the 'thirties he was closely associated with psychologists who were studying personality, and with anthropologists, especially Margaret Mead and Clyde Kluckhohn. These influences resulted in his advancing some modifications of standard psychoanalytic theory, which he put forward in a number of papers, and in a book Childhood and Society, published in 1950. Prominent among the themes developed in that book was ego-identity. Paul Schilder had emphasized that each individual's ego is constant, unchanging and unitary: it has a sense of personal past, present and future. Erikson put the same point, with more detail and supporting evidence drawn from anthropological and social inquiries. Adapting William Harvey's biological term "epigenesis" to the development of personality in its successive phases, he emphasized that the feeling of identity emerges as a consequence of complex adjustments, carried through infancy, childhood, adolescence and adult life. Each phase presents a crisis, calling for adaptation which will harmonize new libidinal forces with new demands made by society.

This theme is reiterated in the book under review, and its application to special groups, such as migrants confronted with the formidable task of striking new roots, is brought into the centre of the picture. Erikson sees identity as a sense of being one with one's self and with the role society casts one for, within social class, political status, or culture. The immigrant, and particularly the refugee, is far more vulnerable than firmly settled people to mishaps in the process of being "identified as a circumscribed individual in relation to a predictable universe which transcends the circumstances of childhood". This matter is explored in the chapter on "Identity and Uprootedness in our Time"; but the theme of identity pervades the whole volume and leads to repetition.

The book comprises six lectures, expanded or in their original form, dealing respectively with Freud, clinical evidence, uprootedness, the cycle of generations, historical actuality, and the golden rule. The last chapter is the one that mainly accounts for the title-page allusion to ethical implications: it was addressed to an Indian audience, and pointed to the affinity between Erikson's concept of epigenesis and the Hindu concept of the life cycle with its four intrinsic goals and its stages on the way to perfection. This lecture, like the first three, was delivered before audiences who could not be assumed to have an intimate understanding of Freudian theory; whereas tho other two chapters, on the cycle of generations and historical actuality, were intended for psychoanalysts and make greater demands on the receptiveness of the nonanalyst reader.

The defects of the book lie in its tendency to oversimplification, its reliance on argument and personal interpretation instead of evidence, its repetition of the central theme, and its uninviting style-which has fallen off greatly since Prof. Erikson wrote Childhood and Socisty. In the earlier book he apologized, for example, for creating the ugly term "generativity", but in the present book there is no such self-criticism: "modern man, forced to limit his fertility, is apt to consider the matter of procreative involvement resolved by the technical possibility of making a conscious choice in the matter of fertilization. For such choice, men must be readied. Yet an ever so 'safe' love life, if accompanied by a mere avoidance of offspring and a denial of generativity could be, in some, as severe a source of inner tension as the denial of sexuality itself has been". Some passages are almost impenetrable; for example, "the study of life histories confirms a far-reaching sameness in men and women in so far as they express the mathematical architecture of the universe, the organization of logical thought, and the structure of language".

In spite of these failings, the book contains stimulating and informative material. This is especially true of the illuminating chapter on the nature of clinical evidence, where the-or perhaps a-psychoanalytical standpoint towards evidence is carefully explained.

\section{Aubrey Lewis}

\section{Mathematical Analysis}

A Special Course. By G. Ye Shilov. Translated by J. D. Davis. English translation edited by D. A. R. Wallace. (International Series of Monographs in Pure and Applied Mathematics, Vol. 77.) Pp. xii +485 . (London and New York: Pergamon Press, Ltd., 1965.) 80s. net.

THIs book contains a considerable amount of mathematics which is almost all at the final year first degree or postgraduate level. The theory of linear spaces is used to introduce such diverse material as the calculus of variations, integral equations and other ideas of functional analysis. The Lebesgue and Stieltjes integrals are treated at length, the former being introduced by the scheme proposed by F. Riesz.

There are many results, all of which have clear proofs, including, for example, theorems giving representations for: (1) a continuous linear functional on the space of functions continuous on a closed interval ; (2) an absolutely monotonic function; (3) a function analytic in the open unit disk and having non-negative real part there; (4) a continuous, positive definite function.

At the conclusion of each chapter there is a short historical note. The whole is well translated, quite readable and to be recommended as an introduction to the ideas of modern analysis.

R. L. Perry

\section{Techniques of Oscillographic Polarography}

By Robert Kalvoda. Second edition, completely revised and enlarged. Pp. 214. (Amsterdam, London and New York: Elsevier Publishing Company; Prague: SNTL, 1965.) $60 s$.

Few, if any, of the physical techniques have spread their influence into the different branches of chemistry more than polarography, or have developed so many variations. Modern chemistry has at its call d.c., square wave, pulse and a.c. oscillographic polarography, to mention only some. The present book deals with the last of these and it would be difficult to find a more authoritative writer, for Dr. Kalvoda, working with Professor Kheyrovsky, has done much to establish oscillographic polarography.

Most of the examples of the application of the technique given in this book are taken from analytical chemistry, but this should not deter those of other chemical persuasions as there is much of interest for all.

The introduction describes compactly the principles of the method, electrode processes which can be followed by it, choice of base solutions, electrodes and the necessary apparatus. However, the substance of the volume is Chapter 2, which deals with applications of the technique. Something for virtually everyone is included: inorganic substances, organic compounds, measurements of reaction kinetics, applications in industrial hygiene and toxicology. 